\title{
Efectos sobre el perfil metabólico, el índice de masa corporal, la composición corporal y la comorbilidad en adolescentes con obesidad mórbida, que han fallado al manejo conservador para bajar de peso, operados de manga gástrica laparoscópica. Reporte del primer grupo de cirugía bariátrica pediátrica en México
}

\author{
Jaime Nieto-Zermeño ${ }^{*}$, Ricardo Ordorica Flores ${ }^{1}$, Blanca Del Río-Navarro², Betzabé Salgado-Arroyo ${ }^{3}$ y \\ Jorge Mario Molina-Díaz \\ ${ }^{1}$ Departamento de Cirugía Pediátrica; ${ }^{2}$ Departamento de Inmunología y Alergia; ${ }^{3}$ Departamento de Nutrición; ${ }^{4}$ Departamento de Endocrinología. \\ Hospital Infantil de México Federico Gómez, Ciudad de México, México
}

\section{Resumen}

Introducción: La obesidad es una pandemia mundial; 3 de cada 10 adolescentes en México la padecen. En la obesidad mórbida, el manejo conservador es un fracaso y la cirugía bariátrica es el único tratamiento útil. No hay experiencia en México y los reportes internacionales son aislados. Método: Análisis de las repercusiones metabólicas, efecto sobre la comorbilidad y complicaciones, en adolescentes con obesidad mórbida operados con manga gástrica de 2011 a 2015. Resultados: De 54 pacientes con obesidad mórbida, 10 cumplieron criterios; 5 varones; edad de $14.3 \pm 1.4$ años; peso de $117.3 \pm 15.2 \mathrm{~kg}$; índice de masa corporal basal de $43.71 \pm 4.1$; cintura de $130.3 \pm 6.8 \mathrm{~cm}$; 8 con dislipidemia; 6 con síndrome metabólico; 5 con esteatosis hepática; 4 con hipertensión; 3 con diabetes tipo 2. Complicaciones: 2 atelectasias, 1 fístula, 1 neumonía, 1 colelitiasis; ninguno con carencia nutricional. Disminución del peso al año del 33\% y a los 2 años del $23 \%$. Disminución de la grasa del 22.4\%. Aumento de agua corporal del 36.1\%. Pérdida del exceso de peso significativa $p=0.001$. A los 2 años, remisión de esteatosis grave y de diabetes, el perfil lipídico mejoró, la hipertensión arterial persistió solo en uno. Discusión: Lo más destacado es la mejoría o curación de la comorbilidad, con curación de la diabetes, la hipertensión y la dislipidemia, que dará mejor pronóstico a estos niños. Conclusiones: La manga gástrica es útil en adolescentes con obesidad mórbida, con mejoría de la comorbilidad grave. Se requiere un mayor seguimiento.

PALABRAS CLAVE: Cirugía bariátrica en niños. Manga gástrica en adolescentes. Repercusiones metabólicas. Comorbilidad en obesidad mórbida.

\section{Abstract}

Introduction: Obesity is a world pandemic; in Mexico 3 out of 10 adolescents suffer from it. Conservative management of morbid obesity is not effective and bariatric surgery is the only useful therapy. International pediatric bariatric surgery series are scarce and in Mexico there is limited experience. Method: We analyze the metabolic repercussions, comorbidities and complications in our series of Mexican adolescents with morbid obesity who underwent a gastric sleeve between 2011 and 2015. Results: 54 morbid obese adolescents were included, 10 fulfilled criteria for surgery. 5 were male, mean age $14.3 \pm$ 1.4 years, weight $117.3 \pm 15.2 \mathrm{~kg}$, initial body mass index $43.71 \pm 4.1$, waist $130.3 \pm 6.8 \mathrm{~cm}, 8$ dyslipidemia, 6 metabolic syndrome, 5 liver steatosis, 4 hypertension, 3 type-2 diabetes. Complications: 2 atelectasis, 1 fistula, 1 pneumonia, 1 choleli-

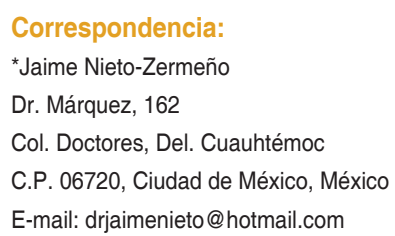

Fecha de recepción: 16-08-2018 Fecha de aceptación: 20-08-2018 DOI: 10.24875/GMM.18004573
Gaceta Med Mex. 2018;154(Suppl 2): S22-S29

Disponible en PubMed www.gacetamedicademexico.com 
thiasis; no patient developed nutritional deficits. Weight loss of 33\% one-year after surgery and $23 \%$ at 2 years. Body fat decreased $22.4 \%$ and water content increased $36.1 \%$. Excess weight loss was significant $p=0.001$. Two-years after surgery severe steatosis and diabetes had subsided, lipid profile improved and only one patient remained hypertensive. Discussion: Our results demonstrate that all serious comorbidities associated to morbid obesity were improved (diabetes, dyslipidemia and hypertension) and will confer a better prognosis for these children. Conclusions: Gastric sleeve is useful in morbid obese adolescents. Longer follow-up is needed.

KEY WORDS: Bariatric surgery in children. Gastric sleeve in adolescents. Metabolic repercussions. Comorbidity in morbid obesity.

\section{Introducción}

La obesidad afecta a millones de individuos en todo el mundo. Es considerada como una verdadera «pandemia", con múltiple comorbilidad asociada, que pone a prueba a todos los sistemas de salud y reduce las expectativas de vida, particularmente en caso de obesidad mórbida. ${ }^{1-4}$

Desde finales del siglo xx, el predominio más alto del exceso de peso de la niñez se ubicó en Europa Oriental, Oriente Medio y España. ${ }^{5,6}$ En los EE.UU., la prevalencia se ha incrementado dramáticamente en adolescentes entre 12 y 19 años. Los datos de la $\mathrm{Na}$ tional Health Examination Survey NHANES de $2017^{7}$ muestran que la obesidad en este grupo de edad, en 1999 , era del $14.8 \%$ y aumentó al $20.6 \%$; es decir, uno de cada cinco adolescentes era obeso. Esta situación es conocida y viene siendo analizada desde hace varias décadas. ${ }^{8,9}$

La Organización Mundial de la Salud destaca la importancia del sobrepeso y la obesidad al informar de que en la actualidad hay en el mundo aproximadamente 340 millones de niños y adolescentes en estas condiciones. ${ }^{10}$

En México, la Encuesta Nacional de Salud y Nutrición (ENSANUT) del año 2012 reportó un incremento en la prevalencia de sobrepeso y obesidad del $33.2 \%$ en el grupo de 12 a 19 años, con mayor afectación del sexo femenino (35.8\%); aumento que ha continuado a pesar de los esfuerzos tanto de los profesionales de la salud como de las autoridades y los especialistas en salud pública, ${ }^{11}$ ya que en la ENSANUT 2016 MC la prevalencia combinada de sobrepeso y obesidad fue del $36.3 \%$, con un aumento de 1.4 puntos porcentuales en comparación con 2012, y fue mayor en zonas urbanas que en zonas rurales, pero este último incrementó un $2.7 \%$ su prevalencia en comparación con 2012.12

El riesgo de la obesidad radica en las complicaciones, que incluyen edad ósea aumentada que afecta la talla final adulta, menarca precoz, alteraciones emocionales (depresión, baja autoestima), hiperlipidemia, hipertensión arterial, esteatosis hepática, problemas ortopédicos, aumento del gasto cardiaco, diabetes mellitus tipo 2, alteraciones en la función pulmonar como la apnea del sueño, asma, disfunción eréctil, etcétera. ${ }^{13-20}$

La obesidad se acompaña de factores de riesgo cardiovascular o de diabetes mellitus tipo 2, que conforman el síndrome metabólico. De acuerdo con la Federación Internacional de Diabetes, en niños y adolescentes, tres o más de cinco criterios establecen el diagnóstico de síndrome metabólico: obesidad abdominal > 90 percentil para su edad y sexo, colesterol ligado a lipoproteínas de alta densidad (HDL) $<45 \mathrm{mg} / \mathrm{dl}$, hipertensión $>130 / 85 \mathrm{mmHg}$, glucemia $\geq 100 \mathrm{mg} / \mathrm{dl}$, triglicéridos $\geq 150 \mathrm{mg} / \mathrm{dl}$. En el seguimiento de una cohorte de la clínica de obesidad del Hospital Infantil de México Federico Gómez identificamos más de un $20 \%$ de adolescentes con los criterios de síndrome metabólico, y en un estudio de nuestro grupo hallamos que de 1880 niños y adolescentes el síndrome metabólico estuvo presente en más del $30 \%$, superior a lo reportado por la Federación Internacional de Diabetes (22\%). ${ }^{21,22}$

Es alarmante que los adolescentes obesos permanecerán como tales en la edad adulta hasta en el $77 \%$ de los casos, con un riesgo relativo de 4 a 6.5 veces y con una clara reducción de la perspectiva de vida (hasta de 20 años). ${ }^{22}$ Esto es de gran relevancia en los pacientes con obesidad mórbida, ya que el tratamiento convencional multidisciplinario ha sido un verdadero fracaso en todo el mundo, con disminución del exceso de peso en cifras tan bajas como el $2-5 \%$, 10 que los ubica con un mal pronóstico. ${ }^{23-25}$

El fracaso del tratamiento médico en la obesidad mórbida está documentado desde el trabajo clásico de Anderson en 1994, quien en un estudio aleatorizado con 27 pacientes demostró que ninguno con obesidad mórbida perdió peso con el tratamiento 
médico. ${ }^{26}$ El Grupo de Estudio de Obesidad de Suecia reportó desde 1988 ninguna respuesta al manejo conservador en 487 pacientes con obesidad mórbida, y en 2017, en el seguimiento de 129 casos por 15 años, solo el $2 \%$ lograron bajar temporalmente algo de peso con manejo no quirúrgico, lo que ratifica que hoy en día el manejo multidisciplinario no quirúrgico en la obesidad mórbida no funciona. . $^{27,28}$

La cirugía bariátrica es actualmente el único método que permite reducir de manera significativa el exceso de peso en los pacientes con obesidad grave, $y$ dentro de ellos están incluidos los adolescentes con obesidad mórbida tratados con dieta y ejercicio, apoyo psicológico y psiquiátrico, y que no consiguen una reducción significativa del peso excedido, persistiendo la comorbilidad. ${ }^{29,30}$

La cirugía bariátrica en adolescentes ha sido aplicada de manera juiciosa en todo el mundo, pero la experiencia es mucho menor que en adultos, solo de 15 a 20 casos por año ${ }^{31}$ Recientemente se ha creado el Teen LABS (Teen-Longitudinal Assessment of Bariatric Surgery), que incluye ocho centros de excelencia en cirugía bariátrica en los EE.UU. y que reportan 242 adolescentes en 4 años, lo que corresponde aproximadamente a ocho casos al año por hospital; esto evidencia que es una cirugía poco frecuente, pero con resultados superiores a lo que se reporta en adultos, porque son individuos con menos años de evolución y por lo tanto con menos deterioro secundario a la comorbilidad y mejor pronóstico. ${ }^{32}$

La gastrectomía en manga es un método restrictivo para tratar la obesidad mórbida. Fue introducido inicialmente como primer paso para la derivación biliopancreática con switch duodenal, con el fin de reducir el peso inicial y el riesgo de mortalidad en la cirugía definitiva ${ }^{33,34}$ Posteriormente, los resultados a corto plazo reportaron que la pérdida de peso y la mejoría en la comorbilidad con la gastrectomía en manga eran similares a las obtenidas con procedimientos de dos etapas. ${ }^{35}$ Por lo tanto, se considera como una operación única, con relativa simplicidad en la técnica, bajo riesgo de complicaciones y secuelas a largo plazo, en comparación con otros procedimientos más invasivos, y además es el que más reduce el hambre por disminución de la grelina. ${ }^{36}$

A pesar de que se cuenta con algunos estudios pequeños que han demostrado resultados alentadores en términos de pérdida de peso y resolución de la comorbilidad en comparación con otros procedimientos definitivos, no se dispone de ningún seguimiento a largo plazo. ${ }^{36,37}$
El objetivo de este estudio fue evaluar la seguridad y la eficacia de la gastrectomia vertical laparoscópica en manga sobre la pérdida de peso, el perfil metabólico, la comorbilidad y los cambios en la composición corporal en adolescentes con obesidad mórbida.

\section{Método}

\section{Diseño del estudio y lugar}

Es un estudio cuasi experimental en adolescentes con obesidad grave que fueron sometidos a cirugía bariátrica mediante gastrectomia laparoscópica en manga en el Hospital Infantil de México Federico Gómez, del periodo de agosto de 2011 a febrero de 2015. Los pacientes fueron monitoreados después de la operación por el cirujano pediatra, el nutriólogo, el endocrinólogo pediatra, el médico en rehabilitación y el psicólogo, de acuerdo con las necesidades de cada paciente.

\section{Participantes}

Todos los adolescentes de este estudio cumplieron criterios para cirugía bariátrica de acuerdo con las guías de la American Society for Metabolic and Bariatric Surgery. ${ }^{38}$ Es decir, menores de 18 años con índice de masa corporal (IMC) $\geq 35 \mathrm{~kg} / \mathrm{m}^{2}$ con comorbilidad mayor (diabetes mellitus tipo 2, apnea obstructiva del sueño moderada a grave, pseudotumor cerebri, esteatosis hepática grave) o IMC $>40 \mathrm{~kg} / \mathrm{m}^{2}$ con o sin comorbilidad (hipertensión arterial sistémica, resistencia a la insulina, síndrome metabólico, intolerancia a los hidratos de carbono o glucosa de ayuno alterada, dislipidemia); así como con la Norma Oficial Mexicano (NOM) para el Tratamiento Integral del Sobrepeso y la Obesidad, y la Guía de Práctica Clínica: Tratamiento Quirúrgico del Adolescente con Obesidad Mórbida. ${ }^{39,40}$

Los pacientes candidatos a cirugía fueron sometidos a un tratamiento multidisciplinario integral durante 1 año, teniendo evaluaciones psicológicas periódicas. Igualmente, todos recibieron la misma guía dietética, que consistía en bioequivalencias de conformidad con las normas establecidas (NOM-043-SSA2-2005, NOM-008-SSA2-1993 y NOM-015-SSA2-1994). La distribución de nutrientes para el conjunto de la dieta era de $15 \%$ de proteína, $30 \%$ de grasa y $55 \%$ de hidratos de carbono. En quienes se documentó fracaso del tratamiento médico (no lograr una reducción del 
$10 \%$ del peso basal) durante al menos 6 meses controlados por un equipo multidisciplinario, presencia de un cuidador dedicado en la familia, evaluación psicológica, conductual, cognitiva, emocional y psicosocial, fueron considerados para incluirse en el grupo de cirugía bariátrica. ${ }^{41,42}$

\section{Recolección de los datos}

Los datos extraídos para este estudio fueron edad, sexo, peso, talla, IMC, complicaciones transoperatorias y posoperatorias, circunferencia de cintura a los 12 y 24 meses posterior a la cirugía, y porcentaje de masa grasa y agua antes de la cirugía y a los 24 meses de esta. Las determinaciones séricas del perfil metabólico se midieron antes de la cirugía y a los 2 años. Este estudio fue aprobado por el comité de ética y de investigación del Hospital Infantil de México Federico Gómez (Protocolo-HIM/2011/022) y contó con Fondos Federales para su realización.

La obesidad fue definida como un IMC $\geq 95$ percentil para la edad y el sexo de las gráficas de los Centers for Disease Control and Prevention (CDC), y la obesidad grave como un IMC $\geq 120 \%$ del percentil $950 \geq 35 \mathrm{~kg} / \mathrm{m}^{2}$. El exceso de peso fue calculado como la cantidad excedente del IMC que tuvo el niño por arriba del 85 percentil del IMC, porque este es el punto de corte que lo define como sobrepeso. ${ }^{40} \mathrm{El}$ porcentaje de pérdida del exceso de peso fue considerado con la siguiente formula: (IMC inicial - IMC final/IMC inicial) $\times 100 .{ }^{43}$

La talla se midió con un estadiómetro de piso (Seca 206, Seca gmbh, Hamburg, Germany). El IMC se calculó como el peso en kilogramos divido por la talla en metros al cuadrado, y se convirtió en las puntuaciones de desviaciones estándar de la graficas de los CDC para edad y sexo. La circunferencia de la cintura se obtuvo con una cinta métrica flexible Seca ${ }^{\circledR}$, en un punto intermedio entre el borde inferior de la última costilla y la cresta iliaca en espiración profunda. En la consulta previa a la cirugía y a los 2 años de realizarla se midieron la glucosa sérica, la insulina, el colesterol total, el colesterol HDL, el colesterol ligado a lipoproteínas de baja densidad (LDL), los triglicéridos, las transaminasas (aspartato transaminasa [AST] y alanina-aminotransferasa [ALT]) y la hemoglobina glucosilada ( $\mathrm{HbA1c}$ ) en una muestra de sangre venosa tras un ayuno de 12 horas. El porcentaje de la grasa corporal y de agua libre se determinó por impedancia eléctrica con un analizador de bioimpedancia (Modelo 1500 MMD; Bodystat Ltd.).

\section{Definición de comorbilidad y de remisión de la comorbilidad}

La presión arterial fue medida en todas las visitas usando un esfigmomanómetro con un brazalete del tamaño apropiado. La hipertensión arterial se definió como una presión sistólica o diastólica por arriba del 95 percentil para el sexo, la edad y la talla, medida en tres o más visitas. La prehipertensión arterial se definió como una presión sistólica o diastólica entre los percentiles 90 y 95 . Todo lo anterior acorde con el cuarto reporte en la evaluación, el diagnóstico y el tratamiento de la presión arterial elevada del niño y el adolescente. ${ }^{44} \mathrm{La}$ dislipidemia fue establecida por los lineamientos del informe del panel de expertos sobre las directrices para la reducción de riesgo y salud cardiovascular en niños y adolescentes. ${ }^{45}$ La diabetes y la prediabetes se diagnosticaron según los criterios de la American Diabetes Association. ${ }^{46}$ El síndrome metabólico fue establecido mediante los criterios de la Federación Internacional de Diabetes. ${ }^{47}$

El diagnóstico de enfermedad de hígado graso no alcohólica fue realizado con la clasificación de Tominaga, de acuerdo con la ecogenicidad hepática, en tres estadios: a) leve, con mínimo aumento difuso de la ecogenicidad hepática, aspecto normal del diafragma y de los vasos intrahepáticos; b) moderada: aumento leve y difuso de la ecogenicidad hepática con ligera disminución de los vasos intrahepáticos y del diafragma; y c) grave: aumento de la ecogenicidad, pobre penetración del segmento posterior del lóbulo hepático derecho y pobre o nula identificación de los vasos hepáticos y del diafragma. ${ }^{48}$

La resolución de la comorbilidad fue evaluada por la clínica y bioquímicamente en cada vista de seguimiento. La glucosa sérica, el perfil de lípidos en ayuno de 12 horas y la HbA1c se repitieron en todos los pacientes. La remisión de la diabetes mellitus tipo 2 fue definida como una glucosa plasmática en ayuno de manera sostenida por debajo de $126 \mathrm{mg} / \mathrm{dl}$ o $7.0 \mathrm{mmol} / \mathrm{l}$, con HbA1c < 6.5\% sin insulina ni hipoglucemiantes orales. Para la dislipidemia se consideraron unos valores de colesterol LDL $<100 \mathrm{mg} / \mathrm{dl} 0<2.8 \mathrm{mmol} / \mathrm{l}$, y de colesterol $\mathrm{HDL}>40 \mathrm{mg} / \mathrm{dl}$ - $1.2 \mathrm{mmol} / \mathrm{l}$. La remisión de la hipertrigliceridemia fue documentada con valores de triglicéridos dentro de rango normal para la edad y el sexo: $<88 \mathrm{mg} / \mathrm{dl} 0<1.0 \mathrm{mmol} / .^{27}$

\section{Procedimiento quirúrgico}

La técnica quirúrgica realizada fue la gastrectomía en manga por vía laparoscópica, colocando cuatro o 
cinco trocares de acuerdo con las necesidades, a 8-10 cm del reborde costal. Para el acceso inicial se utilizaron aguja de Veres y trocares Endopath Xcel con tecnología Optiview Ethicon. Se siguieron los pasos establecidos en la técnica ${ }^{49}$ y utilizando como férula una sonda de calibre $42 \mathrm{~F}$ se realizó la gastrectomía vertical utilizando grapadoras lineales cortantes Endo-GIA 60 mm (Covidien, New Haven, CT, EE.UU.), iniciando por seguridad con dos cartuchos verdes y continuando con los restantes azules. La línea de grapas fue reforzada con un sujete continuo anclado con sutura de nailon de dos ceros. Se colocó un drenaje de succión cerrada de 10F cerca de la línea de corte. Se mantuvo ayuno absoluto de 3 días, después de los cuales se inició la tolerancia oral con líquido coloreado (Gatorate ${ }^{\circledR}$ Azul), y en caso de duda se realizó un estudio radiológico con medio de contraste hidrosoluble; cuando se verificó buen paso y sin fuga ni filtraciones, se inició la vía oral con líquidos. La dieta blanda fue iniciada al cuarto a sexto día posquirúrgico.

\section{Análisis estadístico}

Se usó la prueba de Kolmogórov-Smirnov para verificar la distribución normal de las variables como edad, peso, talla, circunferencia de cintura, IMC y composición corporal. Las variables continuas se presentan en medias y desviaciones estándar. Las proporciones fueron comparadas con la prueba exacta de Fisher. Los cambios entre antes y después de la cirugía en las mediciones de peso y de composición fueron comparados usando una prueba $t$ pareada, y para las variables continuas no relacionadas se usó una prueba $t$ de Student para muestras independientes. La prueba $d$ de Cohen fue usada para estimar el tamaño del efecto.

Se utilizó el paquete estadístico IBM SPSS versión 21 (IBM Corp., Armonk, NY, EE.UU.) para el análisis de los datos. Se consideró estadísticamente significativa una $p<0.05$.

\section{Resultados}

De una cohorte de 54 pacientes de la clínica de obesidad mórbida del Hospital Infantil de México Federico Gómez fueron operados 13 pacientes, pero solo 10 adolescentes cumplieron con 2 años posquirúrgicos y fueron incluidos en este estudio. El 50\% eran de sexo femenino, la media de edad era de 14.3 \pm 1.4 años, el peso de $117.3 \pm 15.2 \mathrm{~kg}$, la talla de $162.6 \pm 5.7 \mathrm{~cm}$, el IMC basal de $43.71 \pm 4.1 \mathrm{~kg} / \mathrm{m}^{2}, \mathrm{y}$ la circunferencia de cintura de $130.3 \pm 6.8 \mathrm{~cm}$. La
Tabla 1. Características antropométricas, parámetros bioquímicos y comorbilidad

\begin{tabular}{|c|c|c|c|}
\hline \multirow[t]{2}{*}{ Variable } & \multirow[t]{2}{*}{ Preoperatorios } & \multicolumn{2}{|c|}{ Posoperatorios } \\
\hline & & 1.er año & $2 .^{\circ}$ año \\
\hline \multicolumn{4}{|l|}{$N=10$} \\
\hline Edad (años) & $14.3 \pm 1.41$ & & \\
\hline Peso (kg) & $117.3 \pm 15.2$ & $92.01 \pm 12.9$ & $92.9 \pm 11.4$ \\
\hline Talla (cm) & $162.6 \pm 5.7$ & $162.6 \pm 5.7$ & $165.6 \pm 6.7$ \\
\hline IMC $\left(\mathrm{kg} / \mathrm{m}^{2}\right)$ & $44.41 \pm 5.13$ & 33. $25 \pm 3.3$ & $34.0 \pm 3.5$ \\
\hline $\begin{array}{l}\text { Circunferencia de } \\
\text { cintura }(\mathrm{cm})\end{array}$ & $130.3 \pm 6.8$ & $106.5 \pm 10.1$ & $109.3 \pm 9.1$ \\
\hline PAS (mm/Hg) & $115.0 \pm 12.0$ & & $111.3 \pm 11.2$ \\
\hline $\operatorname{PAD}(\mathrm{mm} / \mathrm{Hg})$ & $74.3 \pm 8.5$ & & $68.5 \pm 8.5$ \\
\hline Colesterol total (mg/dl) & $172.3 \pm 16.5$ & & $152.5 \pm 21.9$ \\
\hline Colesterol HDL (mg/dl) & $38.9 \pm 6.2$ & & $50.2 \pm 12.9$ \\
\hline Colesterol LDL (mg/dl) & $102.6 \pm 13.8$ & & $88.0 \pm 22.1$ \\
\hline Triglicéridos (mg/dl) & $163.0 \pm 64.26$ & & $85.8 \pm 30.6$ \\
\hline Glucosa (mg/dl) & $96.7 \pm 14.3$ & & $76.3 \pm 6.78$ \\
\hline Insulina ( $\mu \mathrm{UI} / \mathrm{ml})$ & $32.2 \pm 13.3$ & & $8.17 \pm 2.83$ \\
\hline HOMA-IR & $7.97 \pm 4.0$ & & $1.52 \pm 0.61$ \\
\hline $\mathrm{HbA1c}(\%)$ & $6.25 \pm 0.7$ & & $5.40 \pm 0.7$ \\
\hline AST (UI/I) & $43.75 \pm 14.5$ & & $26.5 \pm 16.3$ \\
\hline $\operatorname{ALT}(\mathrm{UI} / \mathrm{I})$ & $65.6 \pm 22.9$ & & $43.6 \pm 4.3$ \\
\hline
\end{tabular}

Datos expresados en media y desviación estándar.

ALT: alanina-aminotransferasa; AST: aspartato transaminasa; HbA1c: hemoglobina glucosilada; HDL: lipoproteínas de alta densidad; HOMA-IR: Homeostasis Model Assessment Insulin Resistence; IMC: índice de masa corporal; LDL: lipoproteínas de baja densidad; PAD: presión arterial diastólica; PAS: presión arterial sistólica.

presión arterial sistólica tuvo una media de $115 \pm$ $12.07 \mathrm{~mm} / \mathrm{Hg}$, y la presión arterial diastólica de 74.3 $\pm 8.5 \mathrm{~mm} / \mathrm{Hg}$ (Tabla 1).

Como comorbilidad asociada a la obesidad en el preoperativo, el $80 \%$ presentaban dislipidemia (hipertrigliceridemia), el $60 \%$ síndrome metabólico, el 50\% esteatosis hepática grave, el $40 \%$ hipertensión arterial y el $30 \%$ diabetes mellitus tipo 2 . No hubo complicaciones en el periodo intraoperatorio. En el posoperatorio temprano (dentro de los primeros 30 días después de la cirugía) se reportó fístula de la gastrectomía en un paciente (que se resolvió con manejo conservador), atelectasia en dos pacientes y neumonía en un paciente. Durante el segundo año de seguimiento, uno presentó colelitiasis que requirió colecistectomía. Ninguno desarrolló deficiencias carenciales de vitaminas o minerales, ya que se llevó un estricto control de la alimentación y suplementación con multivitamínicos. 
Nieto-Zermeño J, et al.: Efectos metabólicos de la manga gástrica en adolescentes con obesidad mórbida

Tabla 2. Cambios en el peso y en la composición corporal desde el preoperatorio, a 1 y 2 años de seguimiento

\begin{tabular}{|c|c|c|c|c|c|c|c|c|c|}
\hline & \multirow[t]{3}{*}{ Basal } & \multicolumn{3}{|c|}{ Primer año } & \multicolumn{3}{|c|}{ Segundo año } & \multirow{3}{*}{$\begin{array}{l}\text { Tamaño del efecto } \\
\text { ( } d \text { de Cohen) }\end{array}$} & \multirow[t]{3}{*}{$\mathrm{p}$} \\
\hline & & \multicolumn{2}{|c|}{ Seguimiento } & \multirow{2}{*}{$\frac{\text { Cambio }}{\text { (\% de pérdida) }}$} & \multicolumn{2}{|c|}{ Seguimiento } & \multirow{2}{*}{$\frac{\text { Cambio }}{\text { (\% de pérdida) }}$} & & \\
\hline & & (media y DE) & $\begin{array}{l}\text { (unidades } \\
\text { absolutas) }\end{array}$ & & (media y DE) & $\begin{array}{l}\text { (unidades } \\
\text { absolutas) }\end{array}$ & & & \\
\hline IMC $\left(\mathrm{kg} / \mathrm{m}^{2}\right)$ & $44.4 \pm 5.1$ & $33.25 \pm 3.3$ & $11.15 \pm 1-6$ & $25.11 \pm 1.5$ & $34.07 \pm 3.5$ & $10.33 \pm 1.6$ & $23.2 \pm 1.9$ & 2.15 & $<0.001^{*}$ \\
\hline IMC (z score) & $2.7 \pm 0.3$ & $1.81 \pm 5$ & $0.88 \pm 0.4$ & $0.32 \pm 0.12$ & $1.9 \pm 0.4$ & $0.80 \pm 0.2$ & $0.30 \pm 0.18$ & 1.36 & $<0.001$ \\
\hline Peso (kg) & $117.3 \pm 15.2$ & $92.0 \pm 12.9$ & $25.3 \pm 9.8$ & $21.56 \pm 7.2$ & $92.9 \pm 11.4$ & $24.4 \pm 8.0$ & $20.7 \pm 7.5$ & 2.25 & $<0.001$ \\
\hline $\begin{array}{l}\text { Circunferencia } \\
\text { cintura }(\mathrm{cm})\end{array}$ & $130.3 \pm 6.8$ & $106.5 \pm 10.1$ & $23.8 \pm 4.7$ & $18.26 \pm 56.5$ & $109.3 \pm 9.1$ & $20.8 \pm 3.7$ & $15.7 \pm 3.6$ & 1.8 & $<0.001$ \\
\hline Agua corporal (\%) & $27.7 \pm 3.3$ & & & & $37.7 \pm 5.7$ & $10.0 \pm 2.4$ & $36.1 \pm 3.0$ & 2.1 & $<0.001$ \\
\hline Grasa corporal (\%) & $62.5 \pm 3.3$ & & & & $48.4 \pm 6.7$ & & $22.4 \pm 2.2$ & 2.3 & $<0.001$ \\
\hline
\end{tabular}

${ }^{*} \mathrm{~d}$ de Cohen basal y 2 años.

DE: desviación estándar; IMC: índice de masa corporal.

El seguimiento del peso y la medición de la composición corporal se realizaron en todos los pacientes de manera basal y a los 2 años de seguimiento. Se midieron el porcentaje de grasa y el porcentaje de agua. Los datos basales, al año y al segundo año se muestran en la Tabla 2. La disminución del peso al primer y al segundo año fue del $33 \%$ y el $23 \%$, respectivamente. La disminución del porcentaje de masa grasa fue del $22.4 \%$ y el aumento del porcentaje de agua fue del $36.1 \%$. Al estratificar por sexos, no hubo diferencia estadísticamente significativa en el sexo masculino $(p=0.06)$ (Figura 1).

Dos pacientes (20\%) permanecieron en obesidad grave a los 2 años de postoperatorio; sin embargo, el IMC estaba por arriba de $45 \mathrm{~kg} / \mathrm{m}^{2}$ y posterior a la cirugía en 37 y $35.6 \mathrm{~kg} / \mathrm{m}^{2}$, por lo que la disminución del porcentaje de grasa y el aumento del porcentaje de agua fueron muy importantes. El porcentaje de pérdida del exceso de peso fue estadísticamente significativo $(p=0.001$ (Figura 2).

En cuanto a la comorbilidad, a los 24 meses se observó remisión completa de la esteatosis grave por ultrasonido, con disminución de la AST a $25.6 \pm 16.3$ y una ALT de $43.6 \pm 4.3 \mathrm{UI} / \mathrm{l}$, control de la diabetes mellitus tipo 2 con suspensión del tratamiento con insulina o metformina, documentándose una $\mathrm{HbA} 1 \mathrm{c}$ de $5.43 \pm 0.17 \%$, y mejora del perfil lipídico, así como de los índices de resistencia a la insulina a través del HOMA-IR, con una media de $1.52 \pm 0.61$, revirtiendo la dislipidemia y el síndrome metabólico en todos los casos. La hipertensión arterial persistió en un paciente, pero se documentó disminución de la dosis y del número de fármacos antihipertensivos.

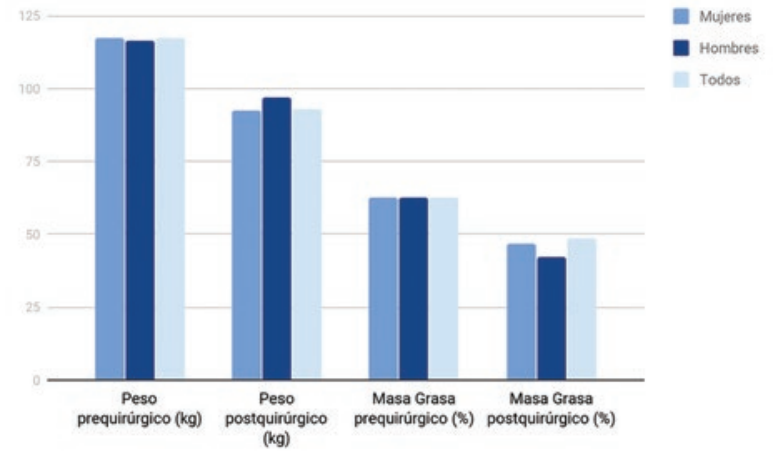

Figura 1. Cambios en el peso y la masa grasa a los 2 años posquirúrgicos.

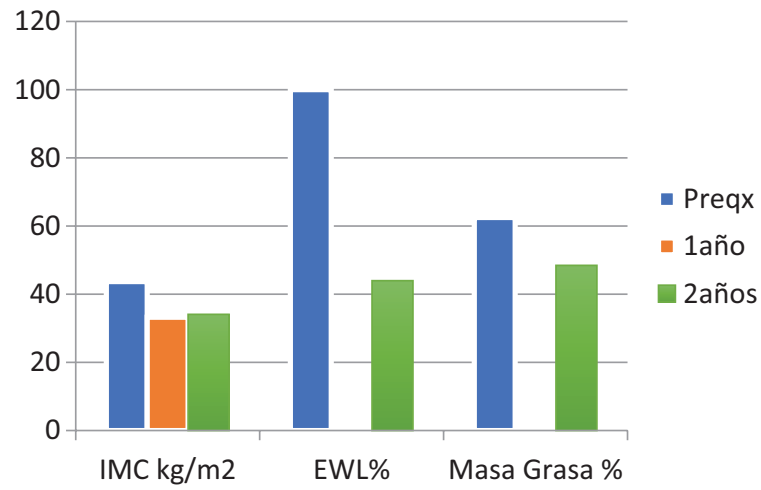

Figura 2. Cambios en el índice de masa corporal y en el porcentaje de exceso de peso a 1 y 2 años de seguimiento. EWL: pérdida de exceso de peso; IMC: índice de masa corporal; Preqx: prequirúrgico.

\section{Discusión}

La obesidad y sus efectos en la salud son la mayor preocupación para los adolescentes hoy en día. La prevalencia de obesidad en los EE.UU. en el grupo de 12-19 años incrementó del 11\% en 1988 al 20\% en 
$2012 .^{50}$ En México se ha observado un incremento similar, del 33.2\% en 2012 al 36.3\% en $2016 .{ }^{12}$

En recientes años ha aumentado el uso de la gastrectomía laparoscópica en manga como tratamiento de la obesidad mórbida, pero la experiencia es limitada en Latinoamérica. ${ }^{31} \mathrm{~A}$ diferencia de lo que ocurre con el tratamiento quirúrgico para el control de la obesidad grave en las personas adultas (sobre el que hay abundante información), en la adolescencia no se ha publicado ningún estudio aleatorizado y controlado, por lo que la información disponible con respecto a los efectos del tratamiento quirúrgico sobre el desarrollo, los aspectos psicológicos y su potencial repercusión sobre el metabolismo es escasa. Por estos motivos, el tratamiento quirúrgico para la obesidad grave en adolescentes debe ser considerado con cautela y después de una evaluación exhaustiva por un equipo multidisciplinario experimentado.

Como se aprecia en nuestros resultados, todos los pacientes mostraron una pérdida de peso inicial, pero a los 2 años de seguimiento se muestra un estancamiento, aunque con mejoría o remisión de la comorbilidad, como ha sido reportado también por otros autores. ${ }^{31,32}$ El cambio en la composición corporal de estos pacientes es evidente, por lo que el incremento en el porcentaje de agua corporal, la disminución de masa grasa y el aumento de la masa muscular pudieran ser la explicación del IMC en rangos no deseables, pero con cambios importantes en el perfil metabólico. Los tres pacientes con diabetes tipo 2 presentaron curación completa, dejando de requerir insulina o metformina, aunque la pérdida de peso no fue la esperada. Es notable también la remisión de la esteatosis hepática y la mejoría de las determinaciones de laboratorio relacionadas con la hepatopatía. La hipertensión arterial desapareció en tres de cuatro, y en uno mejoró, aunque persistió requiriendo menos medicamentos para su control.

\section{Conclusiones}

El tratamiento quirúrgico de la obesidad mórbida en el adolescente es aceptado y realizado en la mayoría de los hospitales americanos y europeos, y es el único método que permite el descenso de peso a largo plazo, mejorando las enfermedades asociadas, la calidad de vida y la autoestima del paciente. En nuestro estudio se observa una pérdida de peso importante, así como un cambio en la composición corporal, pero hay que recalcar la remisión de la comorbilidad, que es el principal factor de riesgo a largo plazo. Sin embargo, es necesario estudios con mayor número de pacientes para poder identificar factores asociados a la pérdida de peso, cambios en el perfil metabólico y cambios en la composición corporal.

\section{Bibliografía}

1. Hedley A. Prevalence of overweight and obesity among US children, adolescents, and adults. JAMA. 2004;291:2847-50.

2. Treadwell J. Meta-analysis of bariatric surgery for pediatric obesity. Ann Surg. 2008;248:763-76.

3. Teen-LABS Study. Cardiovascular risk factors in severely obese adolescents. JAMA Pediatrics. 2015;169:438-44.

4. Fontaine K, Redden D, Wang C, Westfall A, Allison D. Years of life lost due to obesity. JAMA. 2003;289:187-93.

5. Kelishadi R. Childhood overweight, obesity, and the metabolic syndrome in developing countries. Epidemiol Rev. 2007;29:62-76.

6. Sánchez J, Jiménez J, Fernández F, Sánchez M. Prevalence of child and youth obesity in Spain in 2012. Rev Esp Cardiol. 2013;5:371-6.

7. Centers for Disease Control and Prevention. Available at: ww.cdc.gov/ growthcharts. Accessed July 28, 2007

8. Jolliffe D. Extent of overweight among US children and adolescents from 1971 to 2000. Int J Obes Relat Metab Disord. 2004;28:4-9.

9. Flegal KM, Carroll MD, Ogden CL, Johnson CL. Prevalence and trend in overweight among US children and adolescents, 1999-2000. JAMA. 2002;288:1723-7.

10. WHO Multicentre Growth Reference Study Group. WHO Child Growth Standards: length/height-for-age, weight-for-age, weight-forlength, weight-for-height and body mass index-for-age: methods and development. Geneva: World Health Organization, 2006.

11. Gutiérrez J, Rivero D, Shama L, Villalpando S, Franco A, Cuevas L, et al. Encuesta Nacional de Salud y Nutrición 2012. Resultados nacionales. 2. ${ }^{a}$ ed. Cuernavaca, México: Instituto Nacional de Salud Pública; 2013.

12. Hernández M. Encuesta Nacional de Salud y Nutrición de Medio Camino 2016: Resultados ponderados. [Presentación] Instituto Nacional de Salud Pública; 14 de diciembre 2016.

13. WHO Multicentre Growth Reference Study Group. WHO: https://www. who.int/childgrowth/standards/en/Sep 9, 2011.

14. Kimm Y, Obarzanek E. Childhood obesity: a new pandemic of the new millennium. Pediatrics. 2002;110:1003-7.

15. Fagot $A$. Emergency type 2 diabetes mellitus in children: epidemiologic evidence. Pediatr Endocrinol Metabol. 2000;13(Supl 6):1388-405.

16. Strauss-R. Childhood obesity. Pediatr Clin North Am. 2002;49:175-200.

17. Isomaa B, Almgren $P$, Tuomi $T$, Forsen B, Lahi K, Nissen M, et al. Cardiovascular morbidity and mortality associated with metabolic syndrome. Diabetes Care. 2001;24:683-9.

18. Freedman D, Dietz W, Srinivasan S, Berenson G. The relation of overweight to cardiovascular risk factors among children and adolescents: the Bogalusa Heart Study. Pediatrics. 1999;103:1175-82.

19. Teen-LABS Study. Cardiovascular risk factors in severely obese adolescents. JAMA Pediatrics. 2015;169:438-44.

20. Trujillo Palma LE. Síndrome metabólico en pacientes obesos asmáticos y no asmáticos antes y después de orientación nutricional, orientación física y psicoterapia. Tesis de especialidad de Alergia e Inmunología de la Universidad Autónoma de México; 2007. p. 1-60.

21. Del Río-Navarro BE, Velázquez-Monroy $O$, Lara-Esqueda $A$, Violante-Ortiz, R, Fanghanel G, Pérez-Sánchez L, et al. Obesity and metabolic risks in children. Arch Med Res. 2008;39:215-21.

22. Hedley AA. Prevalence of overweight and obesity among US children, adolescents, and adults. JAMA. 2004;291:2847-50.

23. Danielsson P, Kowalski J, Ekblom Ö, Marcus C. Response of severely obese children and adolescents to behavioral treatment. Arch Pediatr Adolesc Med. 2012;166:1103-8.

24. Browne AF, Inge T. How young for bariatric surgery in children? Seminars Ped Surg. 2009;18:176-85.

25. Vannucci A, Wilfley DE. Behavioral interventions and cardiovascular risk in obese youth: current findings and future directions. Curr Cardiovasc Risk Rep. 2012;6:567-78.

26. AndersonT, Backer OG, Stokholm KH, Quaade F. Randomized trial of diet and gastroplasty compared with diet alone in morbid obesity. N Engl J Med. 1984;310:352-6.

27. Karlsson J, Sjöström L, Sullivan M. Swedish Obese Subjects (SOS). An intervention study of obesity, two years follow up of Health-Related Quantity of Life (HRQL) and eating behavior after gastric surgery for severe obesity. Int J Obes. 1988;22:113-26.

28. Sjöström L, Narbro K, Sjöström D, Karason K, Larsson B, Wedel H, et al.; Swedish Obese Subjects Study. Effects of bariatric surgery on mortality in Swedish Obese Subjects. N Engl J Med. 2007;357:741-52. 
29. Carbajo A, Vázquez R, Aparicio R, Luque de León E, Jiménez J, Ortiz J, et al. 12-year old adolescent with super morbid obesity, treated with laparoscopic one anastomosis gastric bypass (LOAGB/BAGUA): a case report after 5-year follow-up. Nutrición Hospitalaria. 2015;31:2327-32.

30. Inge T, Zeller M, Jenkins T, Helmrath $M$, Brandt M, Michalsky M, et al; Teen-LABS Consortium. Perioperative outcomes of adolescents undergoing bariatric surgery: the Teen-Longitudinal Assessment of Bariatric Surgery (Teen-LABS) study. JAMA Pediatr. 2014;168:47-53.

31. Teeple EA, Teich S, Schuster DP, Michalsky MP. Early metabolic improvement following bariatric surgery in morbidly obese adolescents. Pediatr Blood Cancer. 2012;58:112-6.

32. Inge $T$, Courcoulas $P$, Jenkins $M$, Michalsky $P$, Helmrath $A$, Brandt $L$, et al.; the Teen-LABS Consortium. Weight loss and health status 3 years after bariatric surgery in adolescents. N Engl J Med. 2016;374:113-23.

33. Cottam E. Laparoscopic sleeve gastrectomy as an initial weight los procedure for high-risk patients with morbid obesity. Surg Endosc. 2006:20:859-63.

34. Cagner M, Boza C. Laparoscopic duodenal switch for morbid obesity. Expert Rev Med Devices. 2006;3:105-12.

35. Mon Han S, Kim H. Results of laparoscopy sleeve gastrectomy (LSG) at 1 year in morbidly obese Korean patients. Obes Surg. 2005;15:1469-75.

36. Felberbauer F, Langer F, Shakeri-Manesch S. Laparoscopic sleeve gastrectomy as an isolated bariatric procedure: intermediate-term results from a large series in three Austrian centers. Obes Surg. 2008;18:814-8.

37. Sánchez R, Masdevall C, Baltasar A, Martínez C, García R. Short-and mid-term outcomes of sleeve gastrectomy for morbid obesity: the experience of the Spanish National Registry. Obes Surg. 2009;19:1203-10.

38. Michalsky M, Reichard K, Inge T, Pratt J, Lenders C. ASMBS guidelines. ASMBS pediatric committee best practice guidelines. Surg Obes Relat Dis. 2012;8:1-7.

39. Daniels S, Arnett D, Eckel R. Overweight in children and adolescents: pathophysiology, consequences, prevention, and treatment. Circulation. 2005;111:1999-2012.

40. McGill H, McMahan C, Herderick E, Malcom G, Tracy R, Strong J. Origin of atherosclerosis in childhood and adolescence. Am J Clin Nutr. 2000;72:1307-15.

38. Must A, Jaques P, Dallal G, Bajema C, Dietz W. Long-term morbidity and mortality of overweight adolescents. A follow-up of the Harvard Growth Study of 1922 to 1935. N Engl J Med. 1992;327:1350-5.
39. Norma Oficial Mexicana NOM- 008-SSA3-2010, Para el manejo integral de la obesidad.http://www.economia-noms.gob.mx/normas/noms/2010/ 043ssa22013.pdf

40. Guía de práctica clínica: Tratamiento Quirúrgico de la Obesidad Mórbida en el adolescente con obesidad mórbida. Secretaria de Salud 2009. http://www.cenetec.salud.gob.mx/interior/gpc.htm

41. Savoye M, Shaws M, Diura J. Effects of a weight management program on body composition and metabolic parameters in overweight children a randomized controlled trial. JAMA. 2007;297:2697-704.

42. Kelly AS, Barlow SE, Rao G, Inge TH, Hayman LL, Steinberger J, et al.; American Heart Association Atherosclerosis, Hypertension, and Obesity in the Young Committee of the Council on Cardiovascular Disease in the Young Council on Nutrition, Physical Activity and Metabolism, and Council on Clinical Cardiology. Severe obesity in children and adolescents: identification, associated health risks, and treatment approaches: a scientific statement from the American Heart Association. Circulation. 2013;128:1689-712

43. Deitel M, Greenstein RJ. Recommendations for reporting weight loss. Obes Surg. 2003:13:159-60.

44. Falker B, Daniels SR. Summary of the fourth report on the diagnosis, evaluation, and treatment of high blood pressure in children and adolescents. Hypertension. 2004;44:387-8.

45. Expert Panel on Integrated Guidelines for Cardiovascular Health and Risk Reduction in Children and Adolescents; National Heart, Lung, and Blood Institute. Expert panel on integrated guidelines for cardiovascular health and risk reduction in children and adolescents summary report. Pediatrics. 2011;128(Suppl):213-56.

46. American Diabetes Association. Standards of medical care in diabetes-2011. Diabetes Care. 2011;34(Suppl 1):11-61.

47. Zimmet P, Alberti K, Kaufman F, Tajima N, Silink M, Arslanian S, et al. IDF Consensus Group. The metabolic syndrome in children and adolescents - an IDF consensus report. Pediatr Diabetes. 2007:8:299-306.

48. Tominaga K, Kurata J, Chen Y. Prevalence fatty liver in Japanese children and relationship to obesity. An epidemiological ultrasonographic survey. Dig Dis Sci. 1995;40:2002-9.

49. Brethauer DA. Sleeve gastrectomy. Surg Clin N Am. 2011:91:1265-79.

50. Skinner A, Skelton J. Prevalence and trends in obesity and severe obesity among children un the United States 1999-2012. JAMA Pediatr. 2014;168:561-6. 